\title{
Diagnostic significance and potential function of miR-338-5p in hepatocellular carcinoma: A bioinformatics study with microarray and RNA sequencing data
}

\author{
LIANG LIANG $^{1 *}$, LI GAO $^{1 *}$, XIAO-PING ZOU ${ }^{2}$, MENG-LAN HUANG ${ }^{2}$, \\ GANG CHEN $^{2}$, JIAN-JUN LI ${ }^{1}$ and XIAO-YONG CAI ${ }^{1}$
}

${ }^{1}$ Department of General Surgery, The Second Affiliated Hospital of Guangxi Medical University, Nanning, Guangxi 530007;

${ }^{2}$ Department of Pathology, The First Affiliated Hospital of Guangxi Medical University, Nanning, Guangxi 530021, P.R. China

Received March 6, 2017; Accepted November 9, 2017

DOI: $10.3892 / \mathrm{mmr} .2017 .8125$

\begin{abstract}
MicroRNA (miR)-338-5p has been studied in hepatocellular carcinoma (HCC); however, the diagnostic value and molecular mechanism underlying its actions remains to be elucidated. The present study aimed to validate the diagnostic ability of miR-338-5p and further explore the underlying molecular mechanism. Data from eligible studies, Gene Expression Omnibus (GEO) chips and The Cancer Genome Atlas (TCGA) datasets were gathered in the data mining and the integrated meta-analysis, to evaluate the significance of miR-338-5p in diagnosing HCC comprehensively. The potential target genes of miR-338-5p were achieved from the intersection of the deregulated targets of miR-338-5p from GEO and TCGA in addition to the predicted target genes from 12 online software. A protein-protein-interaction (PPI) network was drawn to illustrate the interaction between target genes and to define the hub genes. Gene Ontology (GO) and Kyoto Encyclopedia of Genes and Genomes (KEGG) pathway enrichment analyses were performed to investigate the function of the target genes. From the results, miR-338-5p exhibited favorable value in diagnosing HCC. Types of sample and experiment were defined as the possible sources of heterogeneity in meta-analysis. A total of 423 genes were selected as the potential target genes of miR-338-5p, and five genes were defined as the hub genes from the PPI network. The GO and KEGG analyses indicated that the target genes
\end{abstract}

Correspondence to: Dr Jian-Jun Li or Professor Xiao-Yong Cai, Department of General Surgery, The Second Affiliated Hospital of Guangxi Medical University, 166 Daxue Road East, Nanning, Guangxi 530007, P.R. China

E-mail: lijianjunmail@163.com

E-mail: cxy0771@163.com

*Contributed equally

Key words: miR-338-5p, HCC, GEO, meta-analysis, target genes were significantly assembled in the pathways of metabolic process and cell cycle. miR-338-5p may function as a novel diagnostic target for $\mathrm{HCC}$ through regulating certain target genes and signaling pathways.

\section{Introduction}

Hepatocellular carcinoma (HCC) ranked as the 5th most frequent cancer and was also one of the lethal cancers, particularly in People's Republic of China where liver cancer was the most commonly diagnosed cancer and the most prevalent cause of cancer-related deaths followed by lung, stomach, and esophageal cancers (based on the statistics in 2015) $(1,2)$. Because HCC was often diagnosed at an advanced stage, the prognosis of HCC patients was not optimistic (3). Therefore, a better understanding of the pathogenesis of HCC and a novel target for the early screening of HCC might improve the survival of HCC patients (4).

MicroRNAs (miRNAs) are small non-coding RNAs (18-25 nucleotides in length) that regulate the expression of multiple mRNAs at the post-transcriptional level by suppressing the stability and the translation of mRNAs $(5,6)$. The aberrant expressions of miRNAs was observed in various human cancers, and extensive studies suggested that these deregulated miRNAs had the capacity to distinguish malignant tumors of liver, breast, lung, pancreas and leukemia from adjacent non-tumorous tissue (7-12). miR-338-3p and miR-338-5p originate from an intron of the gene encoding apoptosis-associated tyrosine kinase (AATK). Both miR-338-3p and miR-338-5p are co-expressed because they enjoyed the same promoter together (13). miR-338 was the prvious ID of miR-338-3p, which had been reported in variety of diseases (13-16). miR-338* is one of the members of miR-338 family and usually represented as miR-338-5p (17). As a member of the miRNA family, miR-338-5p was reported to be correlated with the carcinogenesis and progression of several human cancers including gastric cancer (18), colorectal cancer (19), and glioblastoma (20). However, there were limited studies on the clinical significance of miR-338-5p in HCC. Chen et al reported the overexpression of miR-338-5p 
in tumor tissues of the liver and preoperative plasma by miRNA array in Asian patients (21). Whether miR-338-5p is indeed a qualified diagnostic biomarker for $\mathrm{HCC}$ and the underlying molecular mechanism of miR-338-5p in HCC remained unclarified.

Therefore, this study aimed to investigate the diagnostic significance of miR-338-5p in HCC tissues and the molecular mechanism of miR-338-5p in HCC with a combination of meta-analysis and bioinformatics analysis. Our study confirmed the significance of miR-338-5p for the diagnosis of $\mathrm{HCC}$ and might promote the understanding of the molecular mechanism underlying it. The framework of this article was displayed in Fig. 1.

\section{Materials and methods}

The process of study selection. In order to obtain the comprehensive data of the diagnostic value of miR-338-5p in $\mathrm{HCC}$, a thorough search for the related studies was conducted in Gene Expression Omnibus (GEO) dataset and other database including PubMed, Embase, Cochrane, Web of Science, Sinomed, Chinese VIP, Wanfang database and China National Knowledge Infrastructure (CNKI) until December 15, 2016 with the searching strategies: (miR-338 or miRNA-338 or microRNA-338 or miR338 or miRNA338 or microRNA338 or 'miR 338' or 'miRNA 338' or 'microRNA $338^{\prime}$ ) and (malignan" or cancer or tumor or tumour or neoplas" OR carcinoma) AND (hepatocellular or liver or hepatic or HCC). Studies that meet the following criteria were eligible for the meta-analysis: i) Studies evaluated the expression of miR-338-5p for the diagnosis of HCC; ii) the disease of the patients were validated with golden standard; iii) the number of cases were reported in the study; and iv) the sensitivity and specificity of the diagnostic test were available directly or indirectly from the study. The exclusion criteria of the studies were as follows: i) The content of the studies were irrelevant with HCC; ii) the subjects of experiment were not human beings; iii) there was no sufficient data for researchers to directly acquire or calculate sensitivity or specificity of the diagnostic test; and iv) studies were classified as review, meta-analysis, case study or conference note. Moreover, data of the diagnostic value of miR-338-5p in HCC was also downloaded from the cancer genome atlas (TCGA) (https://cancergenome.nih.gov/).

Data extraction and statistical analysis. The following information and data were extracted from the included studies: The ID no. of each GSE chip, first author, year of publication, country, experiment type, platform of each GSE chip, sample number for the experiment group and control group, tissue types, true positivity (TP), false positivity (FP), false negativity (FN) and true negativity (TN).

MetaDiSc1.4 and STATA12.0 were applied for all the statistical analysis. To explore expression of miR-338-5p, the continuous outcomes of GEO and TCGA datasets were calculated with standard mean difference (SMD). The sensitivity (SEN), specificity (SPE), positive likelihood ratio (PLR), negative likelihood ratio (NLR) and diagnostic odds ratio (DOR) of the included studies were pooled with the bivariate meta-analysis model $(22,23)$. The summary receiver operator characteristic (SROC) curve was plotted according to the sensitivity and specificity from each study. The area under the SROC curve (AUC) calculated from the SROC reflected the capacity of miR-338-5p to differentiate HCC patients from non-cancer patients accurately. An AUC value of 0.5 or 1.0 represents a poor or perfect diagnostic value, respectively (24). Additionally, $Q$ test and $\mathrm{I}^{2}$ statistics were employed to assess the heterogeneity between studies. The random-effects model would be used to pool the results if an $\mathrm{I}^{2}$ value was more than $50 \%$ with a P-value $<0.10$; otherwise, a fixed-effects model would be applied $(25,26)$. To identify the source of heterogeneity, the subgroup analysis was conducted based on the number and features of the included studies. With regard to the publication bias, the Deeks' funnel plot asymmetry test was carried out to detect the publication bias, and P-value $<0.05$ was indicative of significance.

The target genes of miR-338-5p. The potential target genes of miR-338-5p came from three sources: The differentially expressed genes from GEO, TCGA and the predicted genes from 12 online software (miRWalk, MicroT4, miRanda, mirBridge, miRDB, miRMap, miRNAMap, PicTar2, PITA, RNA22, RNAhybrid and TargetScan). We firstly searched Gene Expression Omnibus (GEO) datasets for deregulated target genes of miR-338-5p from the mRNA profiling data of HCC samples on December 15,2016. All the GSE chips shared the same platform: GPL570 (Affymetrix Human Genome U133 Plus 2.0 Array). After preliminary screening, 54 studies remained for further selection. Among the 54 studies, Homo sapiens tissue samples instead of cell lines samples were included for further analysis. Finally, $10 \mathrm{HCC}$ datasets (GSE29721, GSE45436, GSE55092, GSE62232, GSE9843, GSE41804, GSE6764, GSE33006, GSE6222 and GSE19665) comprising $431 \mathrm{HCC}$ samples and 198 control samples were chosen for further analysis. Differentially expressed genes (DEGs) between cancer and normal samples of 10 datasets were acquired via GCBI online tool (https://www.gcbi.com. $\mathrm{cn} / \mathrm{gclib} / \mathrm{html} / \mathrm{index}$ ). Fold-change $>1.5$, and a P-value $<0.05$ was set as the threshold for the DEGs. Another database containing high-throughput data: TCGA was also searched. Publicly available miRNA-seq and RNA-seq data of liver HCC was downloaded from the TCGA data portal (December 2016, https://gdc-portal.nci.nih.gov/). Since the TCGA data were a community resource project, additional approval by an ethics committee of our hospital was not mandatory. And the present study adhered to the TCGA publication guidelines and data access policies. From the downloaded data of 377 HCC samples and 50 normal liver samples. R language package DESeq was subsequently used for the calculation of DEGs (Padj $<0.05$ and the absolute $\log 2$ fold-change $>1$ ). As for the predicted genes, selection was based on the condition that they were recorded in more than 4 of the 12 prediction software. The selected qualified target genes from the online software and the validated target genes from miRWalk were considered as the potential target genes of miR-338-5p.

The protein-protein-interaction (PPI) network and validation of target genes. To illustrate the interaction between 


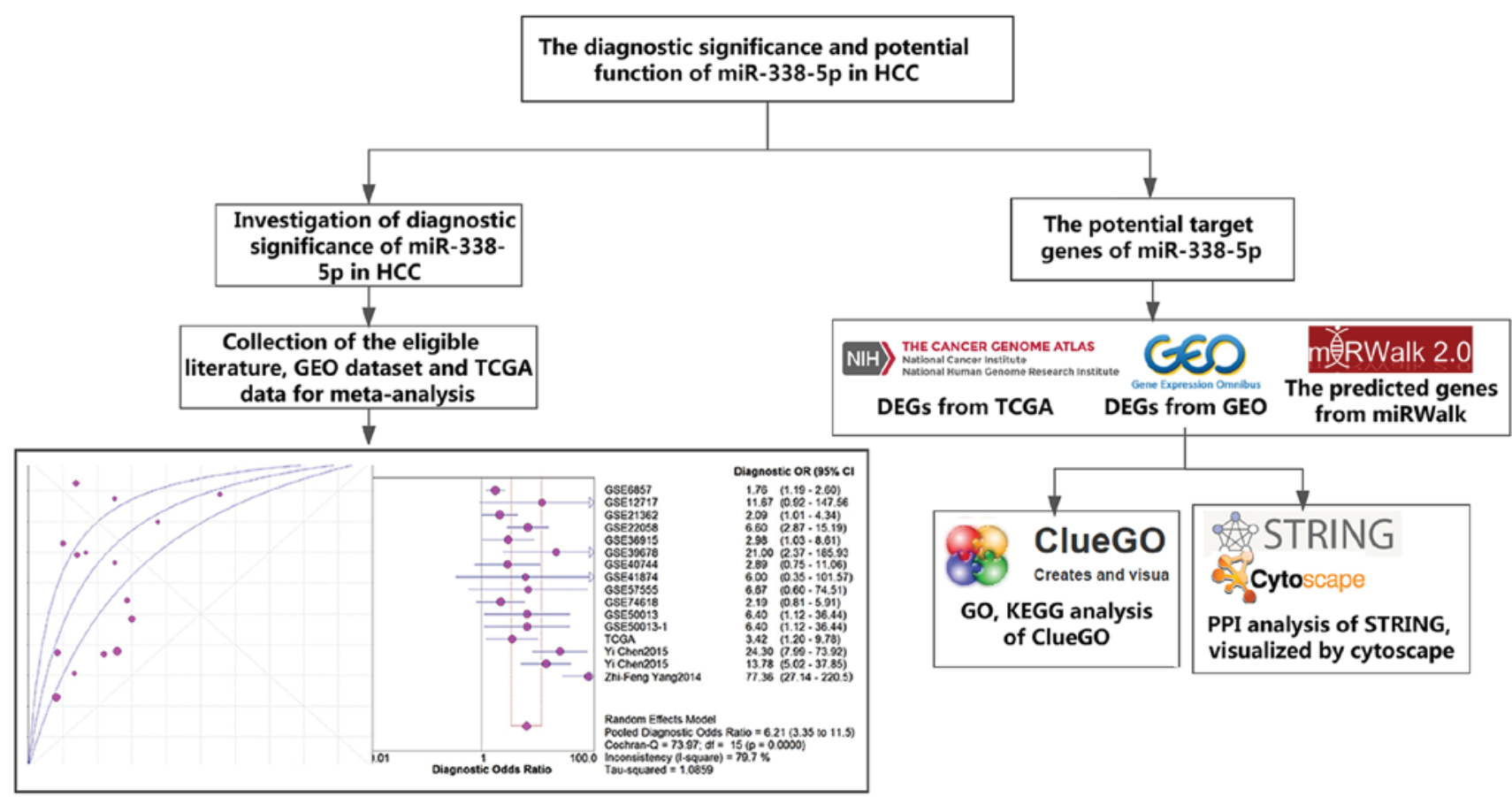

Figure 1. The framework of this study. The framework described the idea of this study.

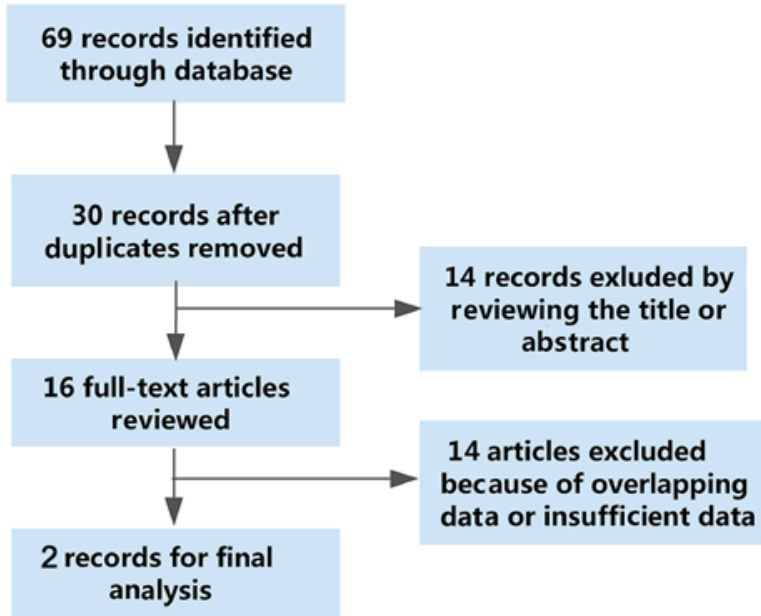

Figure 2. The flowchart: The detailed process of study selection was recorded in the flowchart. After the preliminary screening and full-text scrutinize, three studies were considered eligible studies for the meta-analysis.

the targets of miR-338-5p, a PPI network was drawn by Cytoskape v.5.3.0. The nodes and edges represented target genes and the interactions between target genes, respectively. Hub genes were identified according to the value of degrees of each node. Protein expression of hub gene was validated by The Human Protein Atlas (HPA), an immunohistochemisty (IHC) database (27). Each antibody in the database has been used for IHC staining of both normal and HCC tissues.

The Gene Ontology (GO) and Kyoto Encyclopedia of Genes and Genomes (KEGG) pathway enrichment analysis of the target genes. The GO and KEGG pathway analysis were performed by the BiNGO and ClueGO plug-in unit in Cytoscape v.3.5.0 for the functional annotation of the target genes. Three GO terms including biological process (BP), cellular component (CC) and molecular function (MF) were utilized to identify the enrichment of target genes. P-value $<0.05$ was significant.

\section{Results}

Eligible studies for the meta-analysis. As shown in Fig. 2, the flowchart exhibited the selection and retrieval process of the qualified studies. A total of 69 studies were identified as the initial records, and 30 studies remained after the removal of duplicate records. Then, 14 records were excluded in the preliminary screening of the titles and abstracts of the articles. As a consequence, 16 studies were reviewed in the full text. Among the 16 studies, 14 studies were ineligible due to insufficient data of the diagnostic parameters or duplicate data. Eventually, two studies were enrolled for the meta-analysis. Though two included studies were conducted by the same authors, we failed to validate that the two studies shared the same patient cohorts. Thus, we regard the two studies of Chen et al as two different studies $(21,28)$.

Assessment of the diagnostic value and the integrated meta-analysis. To comprehensively evaluate the diagnostic value of miR-338-5p, we supplemented the literature analysis with GEO data and TCGA data. We searched the GEO dataset with the same searching strategies in literature meta-analysis. Finally, a total of eligible 11 GSE chips were included in our meta-analysis (29-38) (Table I), and the expression level of each study were showed in Fig. 3. With the random-effects model, the forest-plot represented that no significant difference expression was observed between HCC tissue and 


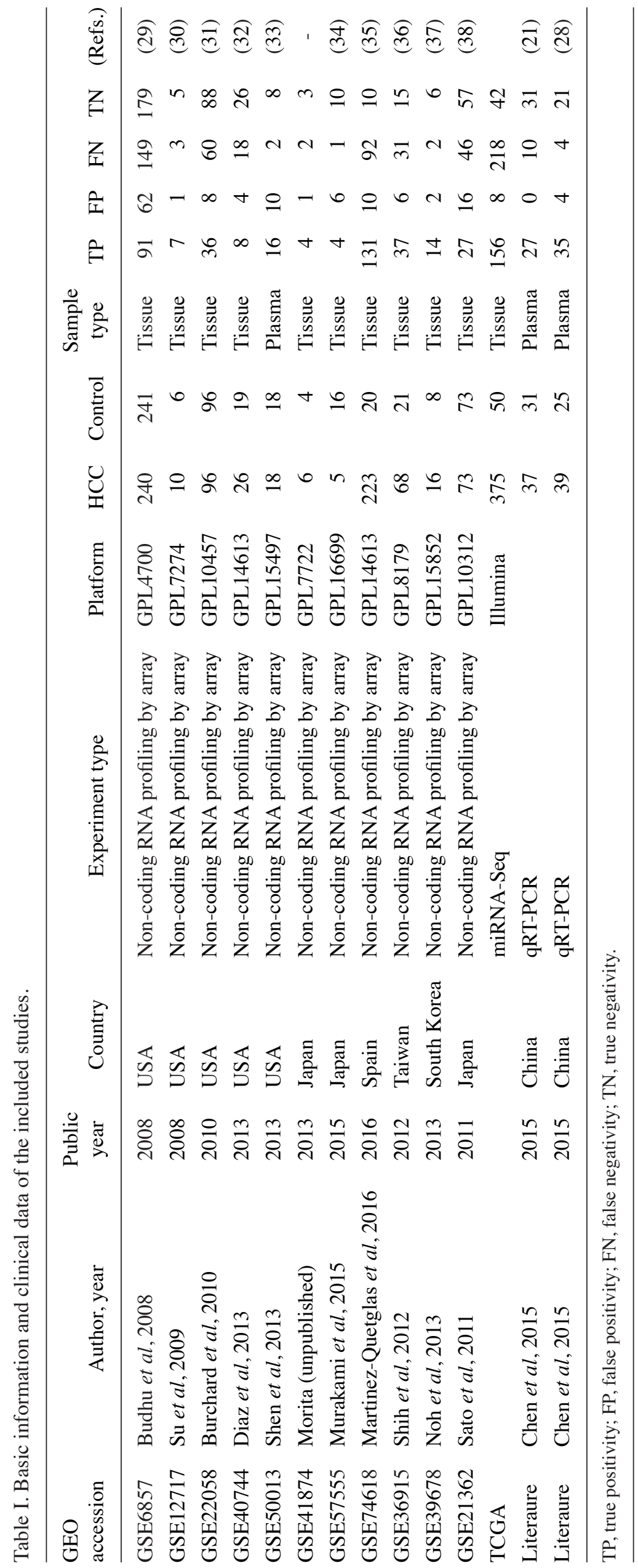



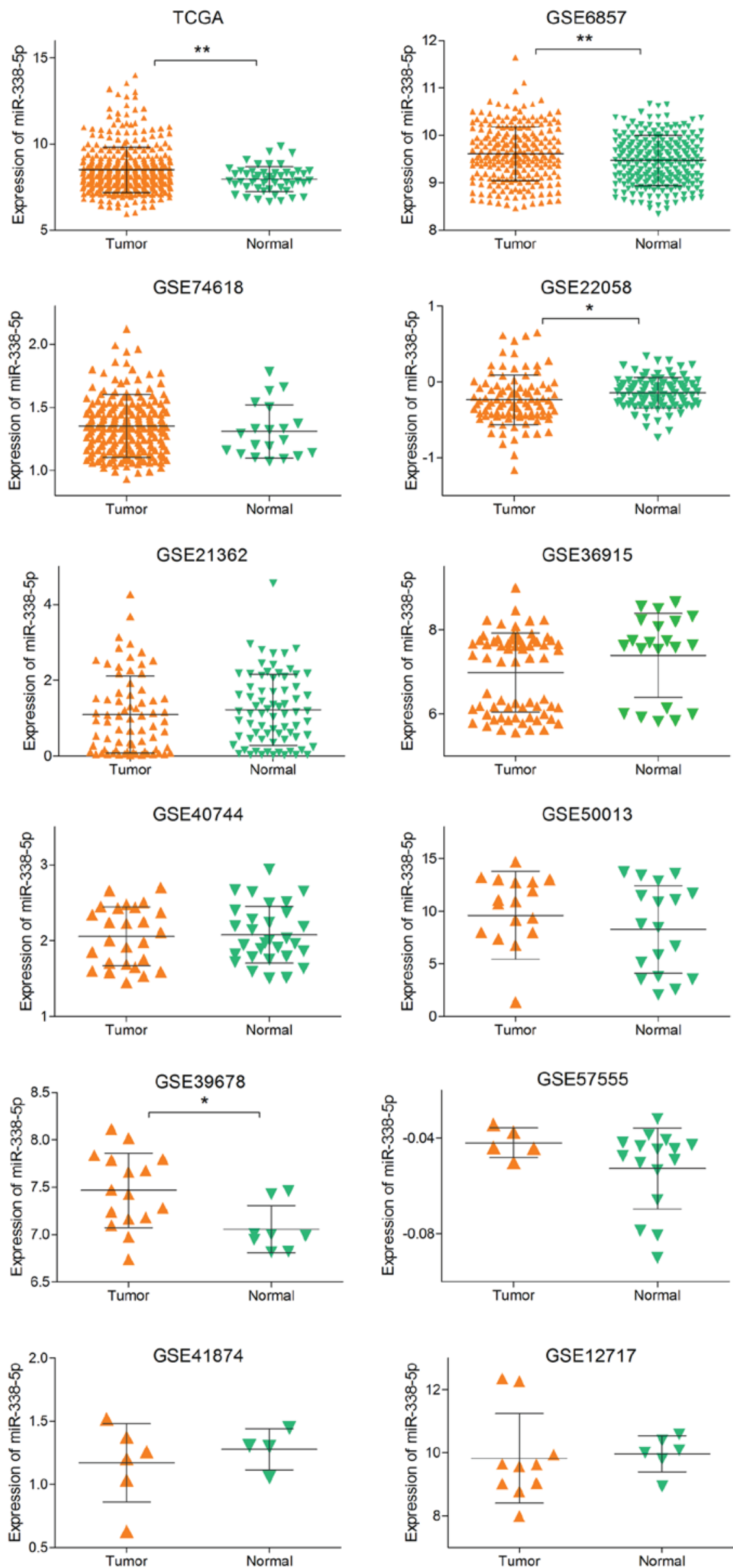

Figure 3. The expression level of each study from TCGA and GEO. Scatter plots were created to illustrate the expression of miR-338-5p in normal tissues and HCC for each study from TCGA and GEO. " $\mathrm{P}<0.05 ;{ }^{* * *} \mathrm{P}<0.01$. TCGA, The Cancer Genome Atlas; GEO, Gene Expression Omnibus; HCC, hepatocellular carcinoma. 


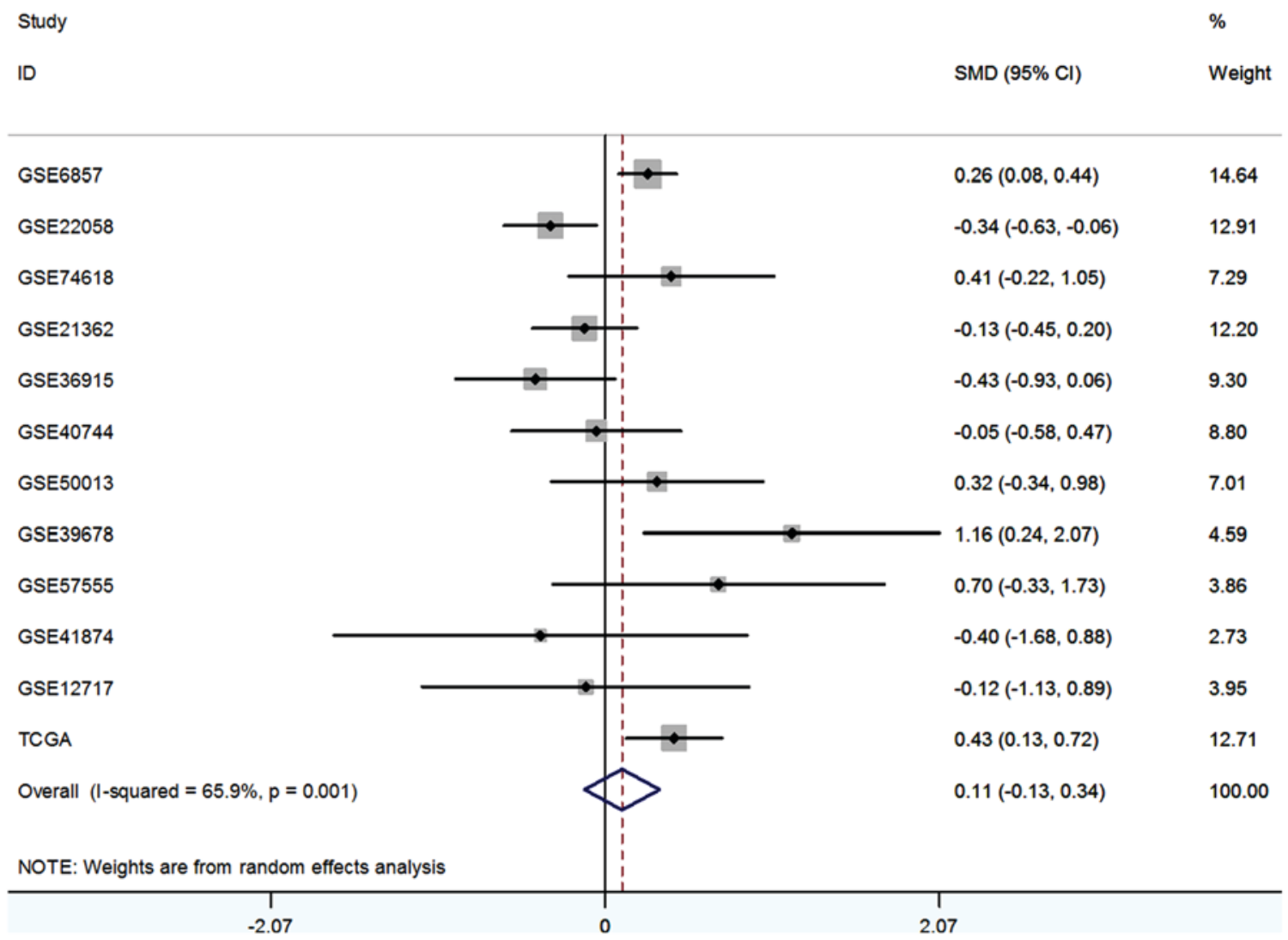

Figure 4. Forest plot of studies calculating SMD of miR-338-5p expression in HCC group compared with control group. SMD, standard mean difference; HCC hepatocellular carcinoma.

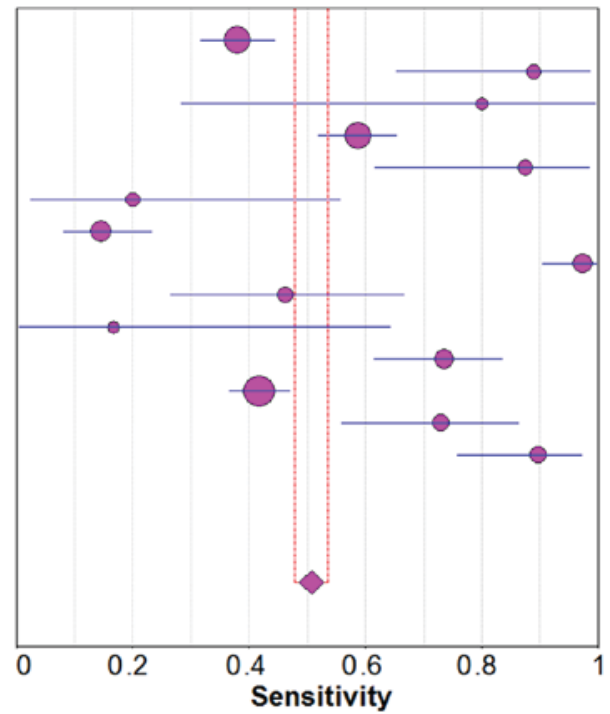

GSE6857 2008

GSE50013 2013

GSE57555 2015

GSE74618 2016

GSE39678 2013

GSE12717 2008

GSE22058 2012

GSE21362 2011

GSE40744 2013

GES41874 2013

GSE36915 2012

TCGA

Yang 2015

Yang 2015

Pooled Sensitivity $=0.51(0.48$ to 0.54$)$

Chi-square $=250.72 ; \mathrm{df}=13(\mathrm{p}=0.0000)$

Inconsistency (I-square) $=94.8 \%$

\section{Sensitivity $(95 \% \mathrm{Cl})$}

$0.38 \quad(0.32-0.44)$

$0.89(0.65-0.99)$

$0.80 \quad(0.28-0.99)$

$0.59 \quad(0.52-0.65)$

$0.88 \quad(0.62-0.98)$

$0.20 \quad(0.03-0.56)$

$0.15(0.08-0.23)$

$0.97 \quad(0.90-1.00)$

$0.46 \quad(0.27-0.67)$

$0.17 \quad(0.00-0.64)$

$0.74 \quad(0.61-0.83)$

$0.42 \quad(0.37-0.47)$

$0.73(0.56-0.86)$

$0.90 \quad(0.76-0.97)$

Figure 5. The pooled SE of the integrated meta-analysis. The pooled SE of the integrated meta-analysis was 0.51 (95\% CI: 0.48-0.54).

normal tissue. The pooled SMD $(0.11,95 \%$ CI: $-0.13,0.34)$ was showed in Fig. 4.

From the chi-square test and $\mathrm{I}^{2}$ test, significant heterogeneity existed in all the pooled effects (SE, SP, PLR, NLR and DOR) between studies (All $\mathrm{I}^{2}>50 \% ; \mathrm{P}<0.05$ ). Therefore, random effects model were employed to estimate the overall SE, SP, PLR, NLR and DOR of all the data. As shown in Figs. 5-9, the SE, SP, PLR, NLR and DOR of all the studies were 0.51 (95\% CI: 0.48-0.54), 0.69 (95\% CI: 0.65-0.73), 1.76 (95\% CI: $1.17-2.66), 0.64$ (95\% CI: 0.52-0.80) and 3.17 (95\% CI: 1.83-5.47). As for the result of SROC, the AUC value of miR-338-5p was 0.691 (Fig. 10). Moreover, the Deeks funnel plot asymmetry test was carried out with Stata 12.0, and no publication bias was detected ( $\mathrm{P}>0.05)$ (Fig. 11). 


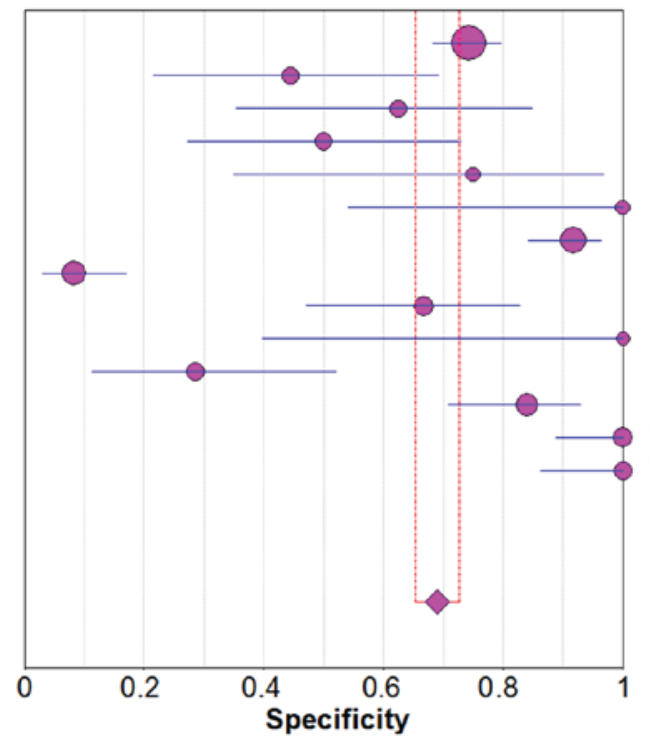

GSE6857 2008

GSE50013 2013

GSE57555 2015

GSE74618 2016

GSE39678 2013

GSE12717 2008

GSE22058 2012

GSE21362 2011

GSE40744 2013

GES41874 2013

GSE36915 2012

TCGA

Yang 2015

Yang 2015

Pooled Specificity $=0.69(0.65$ to 0.73$)$

Chi-square $=229.78 ; \mathrm{df}=13(\mathrm{p}=0.0000)$

Inconsistency (l-square) $=94.3 \%$

Figure 6. The pooled SP of the integrated meta-analysis. The pooled SP of the integrated meta-analysis was 0.69 (95\% CI: 0.65-0.73).

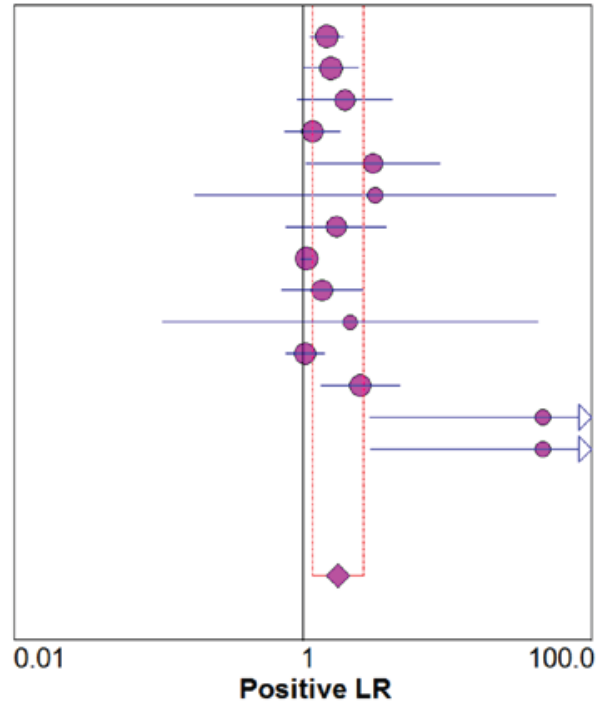

GSE6857 2008

GSE50013 2013

GSE57555 2015

GSE74618 2016

GSE39678 2013

GSE12717 2008

GSE22058 2012

GSE21362 2011

GSE40744 2013

GES41874 2013

GSE36915 2012

TCGA

Yang 2015

Yang 2015

Positive LR (95\% Cl)

$1.47 \quad(1.12-1.92)$

$1.57 \quad(1.01-2.44)$

$1.96 \quad(0.92-4.20)$

$1.17(0.75-1.83)$

$3.07 \quad(1.05-8.97)$

$3.18(0.18-56.95)$

$1.71(0.77-3.79)$

$1.06 \quad(0.98-1.15)$

$1.37 \quad(0.72-2.59)$

$2.14 \quad(0.11-42.52)$

$1.04(0.76-1.41)$

$2.50(1.34-4.68)$

$46.32(2.94-729.65)$

$46.15(2.96-719.93)$

Random Effects Model

Pooled Positive LR $=1.76$ (1.17 to 2.66 )

Cochran- $Q=127.77 ; d f=13(p=0.0000)$

Inconsistency (I-square) $=89.8 \%$

Tau-squared $=0.3890$

Figure 7. The pooled PLR of the integrated meta-analysis. The pooled PLR of the integrated meta-analysis was 1.76 (95\% CI: 1.17-2.66). PLR, positive likelihood ratio.

Now that significant heterogeneity existed between the studies, the subgroup analysis was performed to seek the potential sources of heterogeneity. In the subgroup of sample types, the heterogeneity decreased substantially in the pooling estimates of NLR (49.2\%) and DOR (5.6\%) in the group of tissue. The value of SE, SP, PLR and DOR were obviously higher in the plasma group $(0.83,0.74-0.90 ; 0.86,0.77-0.93$; $14.02,0.08-2,395.96 ; 61.30,3.61-1,040.31)$ than in the tissue group $(0.48,0.45-0.51 ; 0.67,0.63-0.71 ; 1.51,1.08-2.11$; $2.05,1.51-2.77)$ and the value of NLR was notably lower in the plasma group $(0.21,0.11-0.38)$ than in the tissue group (0.81, 0.71-0.92).

With regard to the subgroup of experiment, the heterogeneity decreased substantially in the pooling estimate of DOR (10.3\%) in the microarray group, and declined heterogeneity of SE (0\%), PLR (0\%) and DOR $(0.0 \%)$ were also observed in the group of qRT-PCR. The value of SE, SP, PLR and DOR were obviously higher in the qRT-PCR group (0.82, 0.71-0.90; 1.00, 0.94-1.00; 46.23, 6.60-323.68; $254.42,32.2-2,010.45)$ than in the microarray group $(0.49$, $0.46-0.52 ; 0.66,0.62-0.70 ; 1.51,1.11-2.06 ; 2.15,1.56-2.97)$ and the value of NLR was notably lower in the qRT-PCR group $(0.19,0.08-0.47)$ than in the microarray group $(0.79$, 0.69-0.91). This result confirmed that types of sample and experiment were the possible sources of heterogeneity in this study.

Bioinformatics study of the target genes of miR338-5p. According to the results, a total of 1,698 and 1,798 genes were identified as DEGs targeted by miR-338-5p from TCGA and 


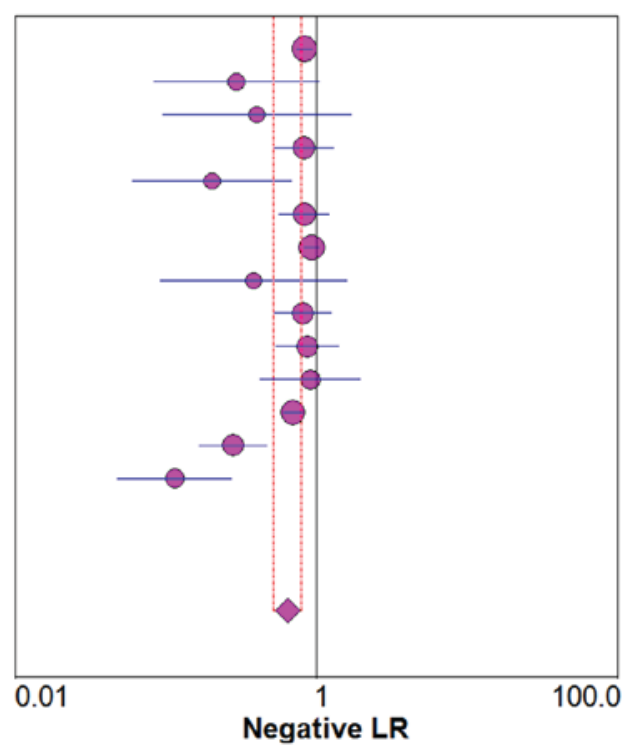

Negative LR $(95 \%$ C

GSE6857 2008

GSE50013 2013

GSE57555 2015

GSE74618 2016

GSE39678 2013

GSE12717 2008

GSE22058 2012

GSE21362 2011

GSE40744 2013

GES41874 2013

GSE36915 2012

TCGA

Yang 2015

Yang 2015

\section{Random Effects Model}

Pooled Negative LR $=0.64(0.52$ to 0.80$)$

Cochran- $Q=69.37 ; d f=13(p=0.0000)$

Inconsistency (l-square) $=81.3 \%$

Tau-squared $=0.0868$

Figure 8 . The pooled NLR of the integrated meta-analysis. The pooled NLR of the integrated meta-analysis was 0.64 (95\% CI: 0.52-0.80). NLR, negative likelihood ratio.

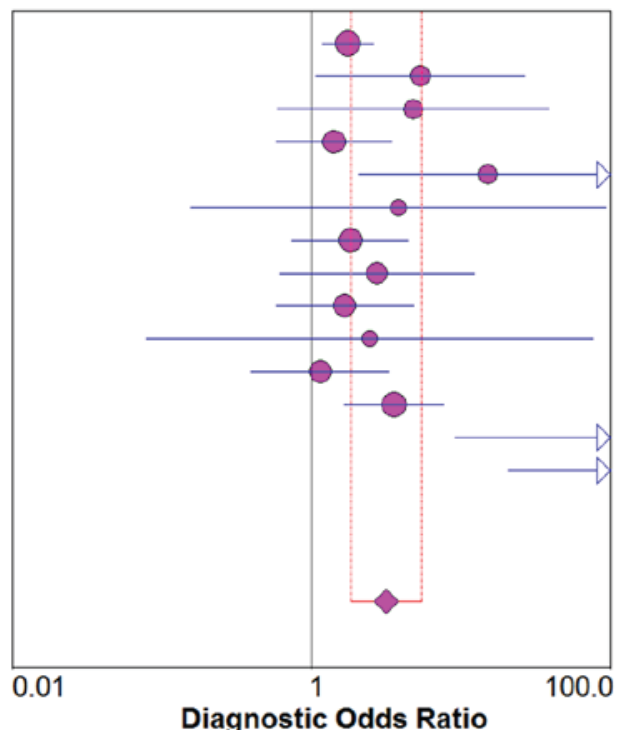

GSE6857 2008

GSE50013 2013

GSE57555 2015

GSE74618 2016

GSE39678 2013

GSE12717 2008

GSE22058 2012

GSE21362 2011

GSE40744 2013

GES41874 2013

GSE36915 2012

TCGA

Yang 2015

Yang 2015

Random Effects Model

Pooled Diagnostic Odds Ratio $=3.17(1.83$ to 5.47$)$

Cochran- $Q=34.23 ; \mathrm{df}=13(\mathrm{p}=0.0011)$

Inconsistency (l-square) $=62.0 \%$

Tau-squared $=0.5159$

Figure 9. The pooled DOR of the integrated meta-analysis. The pooled DOR of the integrated meta-analysis was 3.17 (95\% CI: 1.83-5.47). DOR, diagnostic odds ratio.

GEO, respectively. Additionally, a total of 3,610 predicted target genes that appeared in more than four times of the 12 online software were obtained. Taking the intersection of the DEGs from GEO and TCGA as well as the qualified predicted targets genes, we selected 423 genes for the following bioinformatics analyses (Fig. 12). The PPI network shown in Fig. 13 illustrated the interactions between the target genes of miR-338-5p. There were 147 nodes and 248 edges in the network. Hub genes with a degree values of more than 11 including NCOR1, IGF1, FOXO1, FOS, CDCA8, BUB1B, PCNA, ESR1, BIRC5, MYC and CDK1 were emphasized in red while the remaining were colored in green. To verify that these hub genes are targeted by miR-338-5p, we obtained the immunohistochemical staining of several of the hub genes including NCOR1 and FOXO1 in HCC tissues and normal tissues. As shown in Fig. 14, NCOR1 and FOXO1 were found to have medium staining and moderate intensity in cytoplasmic/menbranous of normal tissues, while a lower staining and weaker intensity of these genes were observed in HCC tissues.

According to the results of GO analysis in cytoskape, the target genes were found to enrich most significantly in the following biological pathways: response to organic substance, response to chemical stimulus and oxoacid metabolic process. As for cellular component and molecular function, target genes mainly assembled in extracellular region part and binding, respectively (Table II; Fig. 15). Moreover, a total of 5 significant pathways were recorded from the KEGG pathway 


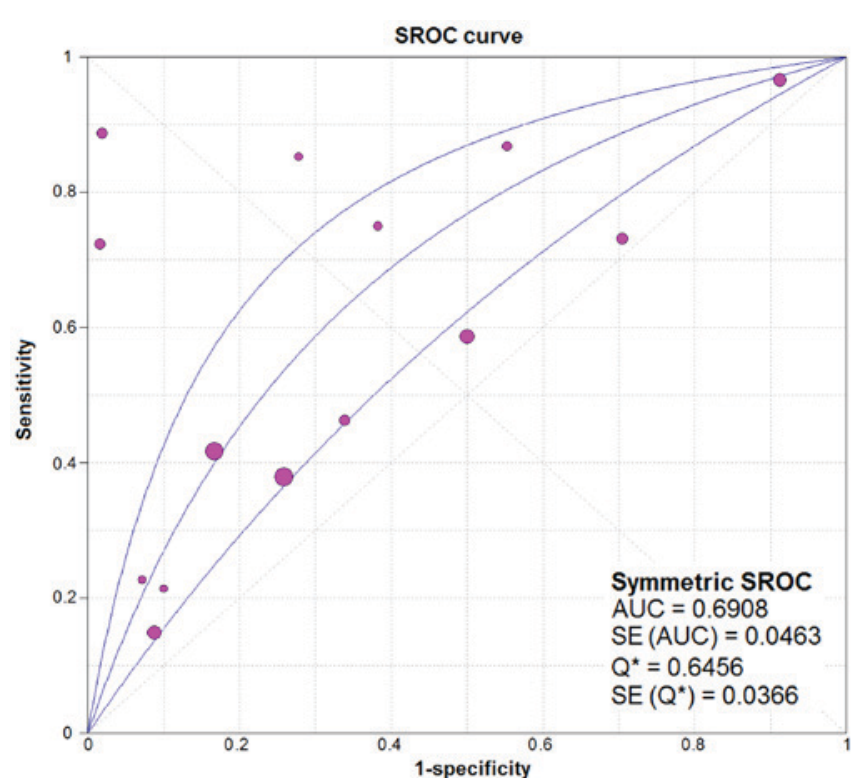

Figure 10. The pooled SROC of the integrated meta-analysis. The pooled SROC of the integrated meta-analysis was 0.691 . SROC, summary receiver operator characteristic.

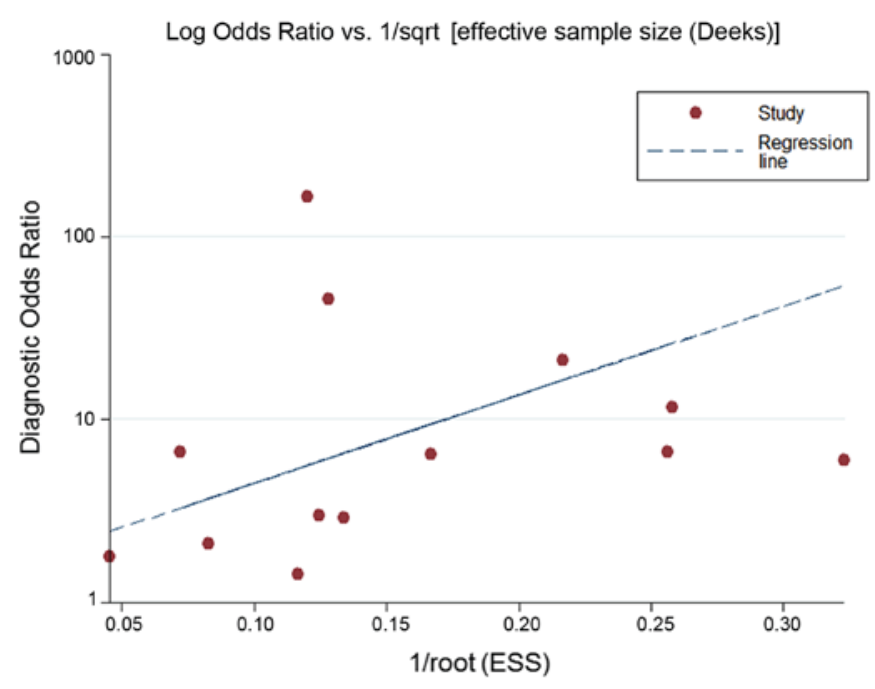

Figure 11. The publication bias. The symmetrical Deeks funnel plot indicated no publication bias.

analysis such as valine, leucine and isoleucine degradation, pathways in cancer and cell cycle (Table III; Fig. 16) were the most significant.

\section{Discussion}

Considerable attention has been attracted to miRNAs as promising diagnostic targets for the early screening of human cancers. Prior to our study, several researches have reported some miRNAs had diagnostic value in HCC. A 3-miRNA panel: miR-92-3p, miR-107, and miR-3126-5p discovered by Zhang et al were claimed to distinguish HCC patients in early stage and HCC patients with low-level AFP from their corresponding controls with high accuracy (39). Additionally, some single miRNAs including miR-21 and

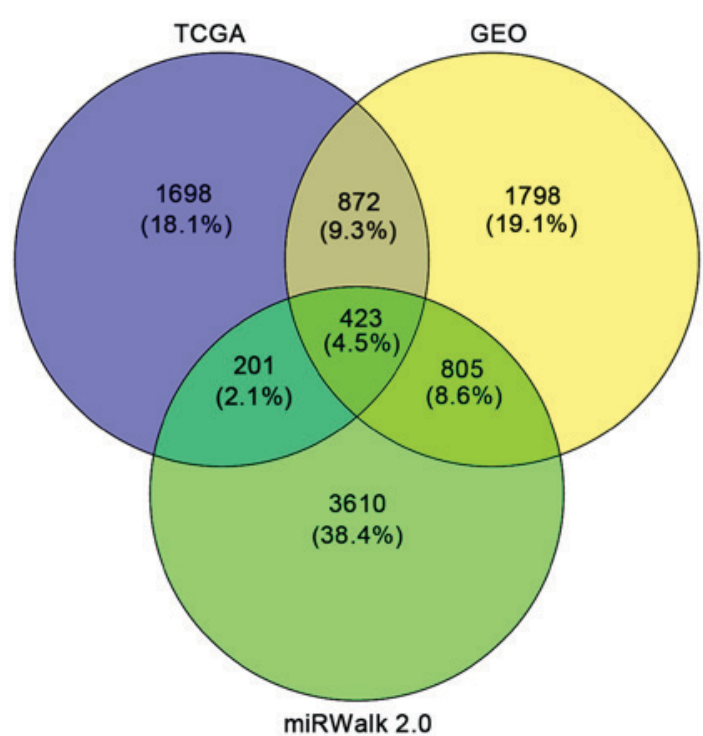

Figure 12. The Venn diagram for the target genes of miR-338-5p. Three circles represented the collected target genes from three different sources (blue for TCGA, yellow for GEO and green for the predicted target genes) There were 1,698, 1,798 and 3,610 potential target genes from TCGA, GEO and online software, respectively. A total of 423 genes were obtained after taking the intersection of the three parts. TCGA, The Cancer Genome Atlas; GEO, Gene Expression Omnibus.

miR-224 also exhibited prominent diagnostic potential for HCC $(40,41)$ and so far, only one study referred to the diagnostic value of miR-338-5p in HCC with the method of miRNA array. Chen et al (21) reported a moderate ability of miR-338-5p to differentiate HCC from liver cirrhosis with the AUC of 0.799 . Furthermore, an extremely strong diagnostic value of miR-338-5p (AUC=0.909) was observed when diagnosing HCC from healthy controls. Despite some advances has been made in exploring the diagnostic capacity of miRNAs for $\mathrm{HCC}$, the diagnostic significance of miR-338-5p in HCC was indefinite, and the relative molecular mechanism has not been elucidated in these studies; therefore our study was the first one to comprehensively assess the diagnostic value of miR-338-5p in HCC with the data from GEO, TCGA and literature as well as to investigate the underlying molecular mechanism through bioinformatics study.

From the meta-analysis result from our collected literature and the integrated meta-analysis, miR-338-5p may serve as a possible diagnostic target for HCC with fair sensitivity and specificity, which enlightened us that miR-338-5p might play an essential role in the occurrence and progression. Previous studies have pointed out that miR-338-5p exerted a tumor suppressive function in a wide range of cancers. In glioblastoma, miR-338-5p was discovered to inhibit the proliferation, invasion and promote apoptosis by targeting EFEMP1 (42); similarly, miR-338-5p significantly attenuated the malignant potential of gastric cancer cells through regulating BMI1 (13). In contrast, miR-338-5p was increased in both blood and tissue of coloreactal cancer (CRC), and represented high area under ROC curve (AUC) of 0.871 . The performance of miR-338-5p indicated that it could be a potential biomarker in CRC (19). A similar trend was observed in CRC compared with HCC. Furthermore, according to the 


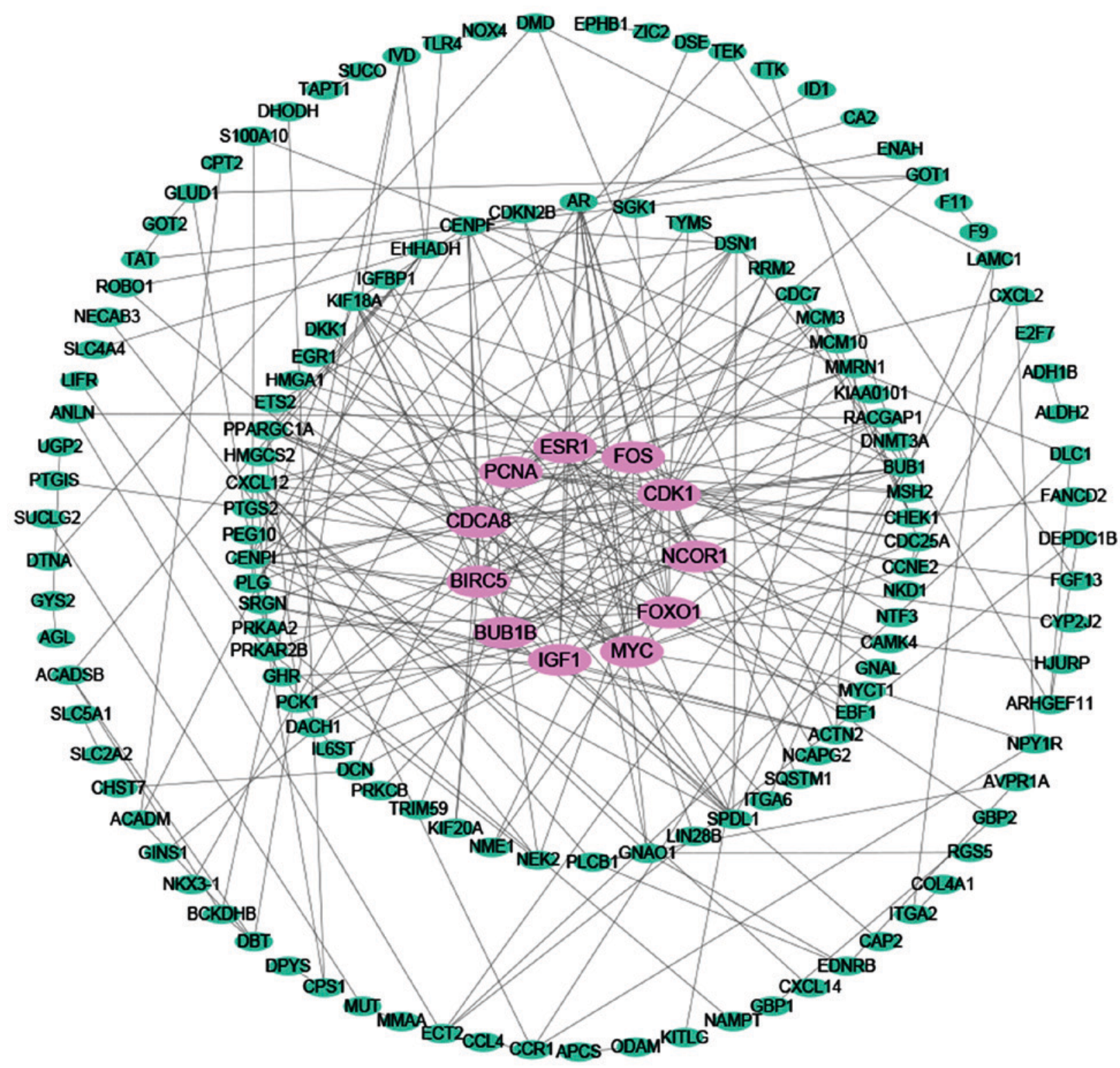

Figure 13. The PPI network of the target genes of miR-338-5p. A total of 147 nodes and 248 edges constituted the network, from which 11 hub genes colored in red were identified according to the value of degrees. PPI, protein-protein-interaction.

subgroup analysis in the integrated meta-analysis, studies with samples from plasma and the method of qRT-PCR were more precise in diagnosing HCC than studies with the controlled conditions, which hinted that the sample type and experiment type may also influence the accuracy of the diagnosis. Although the overall diagnostic ability of miR-338-5p in HCC was the same, which was reflected by the integrated meta-analysis and the meta-analysis from our literature there were still some differences between them. The sensitivity, specificity, diagnostic odds ratio and the area under SROC of the result from the literature meta-analysis were higher than those from the integrated meta-analysis, especially in the evaluation of sensitivity; miR-338-5p showed a poor sensitivity of only 0.51 in the integrated meta-analysis. This might be attributed to the difference in sample type and experiment type as well as the sources of the data. The integrated meta-analysis included GSE datasets from different platforms and TCGA data based on the literature meta-analysis, the samples of which were different. Moreover, due to the limited number of literature, we failed to trace the heterogeneity by carrying out subgroup analysis for our literature meta-analysis. Expanding the sample size was necessary for a more reliable assessment of the diagnostic value of miR-338-5p in HCC.

The results from meta-analysis only provided a superficial hint that miR-338-5p possessed significant diagnostic capacity in $\mathrm{HCC}$ and the molecular mechanism underlying it needed further exploration. Thus, we emphasized on the network and functional analysis of the target genes.

We firstly identified the potential target genes of miR-338-5p and further defined the hub genes from PPI network. The 11 hub genes were assumed to correlate closely with miR-338-5p and play essential roles in the miR-338-5p relevant pathogenesis of HCC. Among the hub genes, CDK1 was important protein for the regulation of cell cycles belonging to the cyclin-dependent kinases family (43). The 

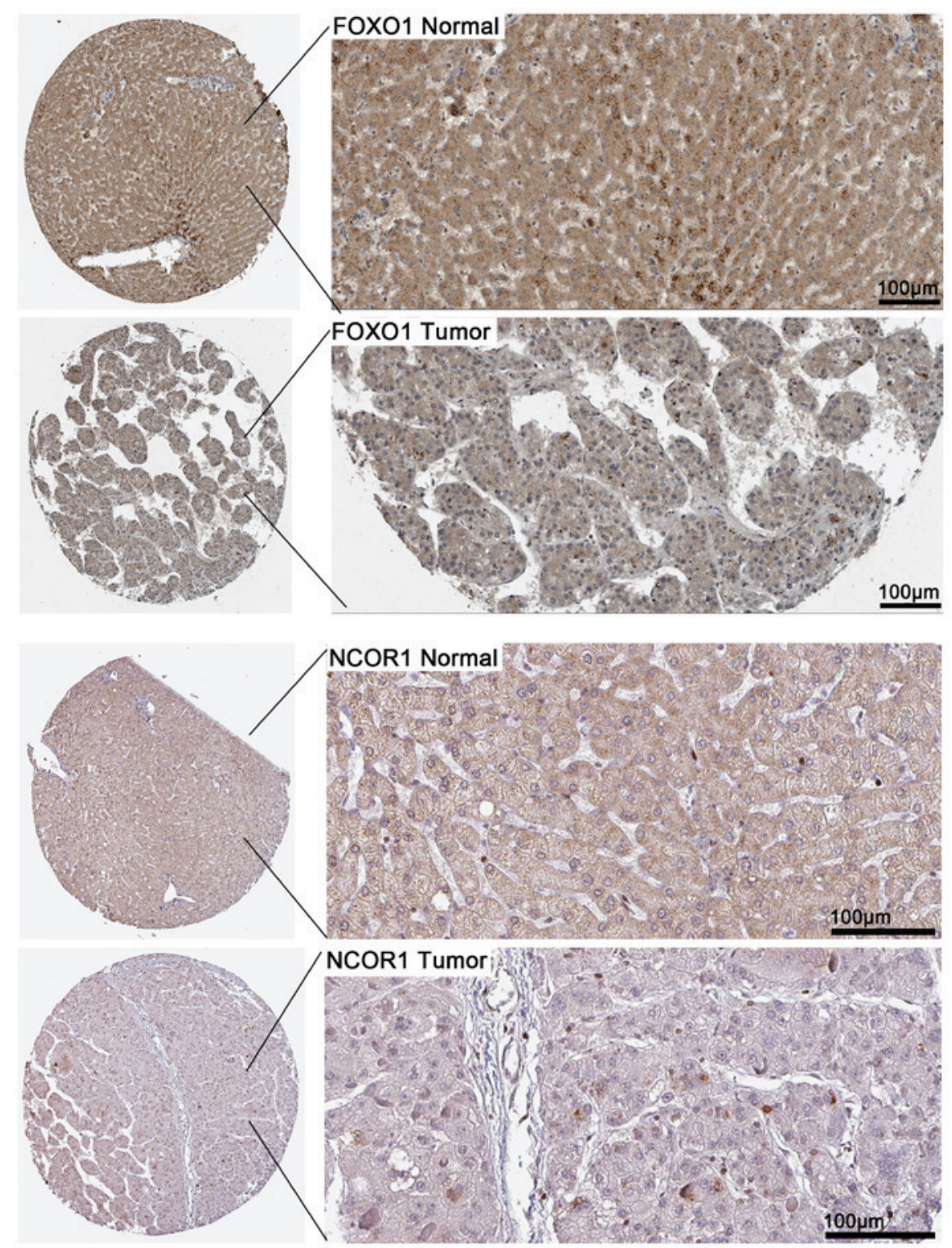

Figure 14. The immunohistochemical staining of NCOR1 and FOXO1 in normal tissues and HCC tissues. According to the immunohistochemical results of these genes in HPA database, NCOR1 and FOXO1 presented medium staining in normal tissues; while a lower staining of these genes were observed in HCC tissues. HCC, hepatocellular carcinoma; HPA, the Human Protein Atlas.

overexpression of CDK1 was detected in various cancers, and a poor prognosis of renal cell carcinoma patients was associated with the high expression of CDK1 and CDK2 (44-48). We hypothesized that CDK1 deregulated by miR-338-5p might promote the deterioration of $\mathrm{HCC}$ by affecting the cell cycle of HCC cells. Apart from CDK1, several hub genes such as MYC, BIRC5, IGF1, NCOR1 and FOXO1 participate in the regulation of a wide range of biological processes including cell proliferation, apoptosis and migration (49-57). These genes were reported to be aberrantly expressed in various cancers (58-62) and they were also involved in the malignant progression of HCC $(49,50,52,63)$. It was conceived that miR-338-5p might interact with these molecules through potential signaling pathways to influence the development of HCC. PCNA, a protein that acted as DNA sliding clamp, was found to engage in DNA duplication and repair with its overexpressed in HCC. Further study was necessary to probe into the association between PCNA and miR-338-5p in HCC.
In this study, we analyzed 11 hub genes protein expression by HPA database. The result indicated that the expression of FOXO1 and NCOR1 was downregulated in HCC and most likely regulated by miR-338-5p.

GO enrichment analysis was indicative of the possible functions of the target genes in $\mathrm{HCC}$ and the results from three GO terms hinted that the target genes were mainly assembled in response to organic substance. Most of the potential functions of the target genes from the GO analysis were accomplished in signaling pathways. Therefore, it is of great importance to investigate the signaling pathways gathered by the target genes of miR-338-5p. From the results of the KEGG pathway analysis, the most significant ten pathways such as pathways in cancer and cell cycle were closely associated with cancer.

Despite the valuable findings acquired from the meta-analysis and bioinformatics study, there were still some limitations in our study. The sample size of our literature was 


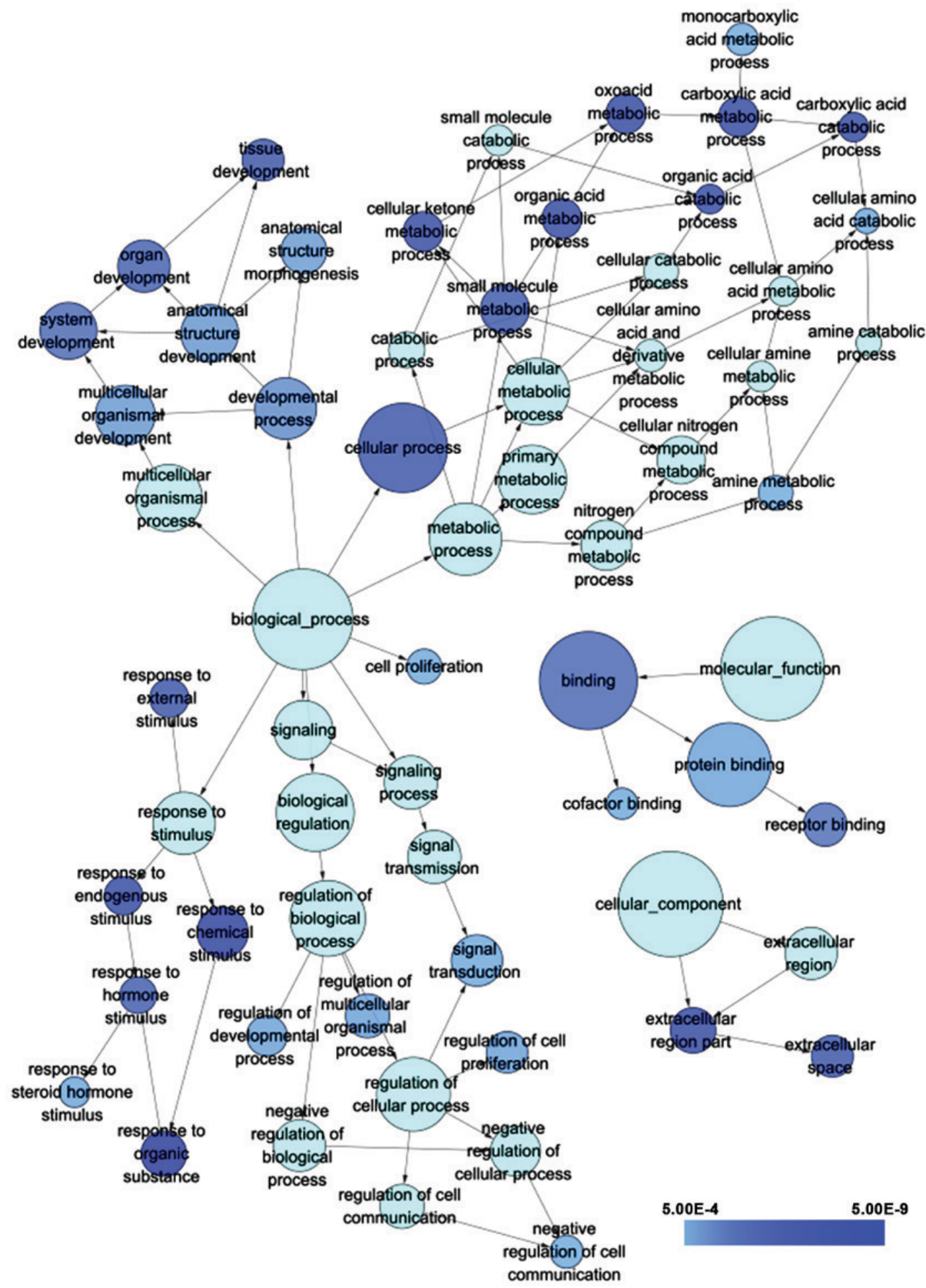

Figure 15. The GO terms of target genes of miR-338-5p. The circles represented different GO terms of BP, CC and MF. The darker the color of the circles presents, the greater the significance the term indicated. The relationship between different GO terms were symbolized by arrows. GO, Gene Ontology; $\mathrm{BP}$, biological process; $\mathrm{CC}$, cellular component; MF, molecular function.

too small for further analysis to identify heterogeneity, which weakened the reliability of our results. Since the samples are from different types, including tissue and plasma, a bias and sensitivity problems might originate from sample types in analysis. Additionally, we only included studies published in Chinese or English, which might cause bias of selection to the meta-analysis. A plausible way to address these issues is to conduct future studies with larger samples and fewer language restrictions to further verify the diagnostic value of miR-338-5p for HCC.

In conclusion, we anticipated that miR-338-5p may serve as a promising diagnostic marker for HCC and miR-338-5p could affect the development of $\mathrm{HCC}$ by targeting certain downstream genes and pathways. The future research will be concentrated on validating the target genes of miR-338-5p and its function in the significant signaling pathways mentioned before. 
Table II. GO functional annotation of the target genes of miR-338-5p from Cytoskape.

\begin{tabular}{|c|c|c|c|c|}
\hline ID & Category & GO term & P-value & Count \\
\hline GO:0006082 & GO_Biological process & Organic acid metabolic process & $3.87 \times 10^{-9}$ & 37 \\
\hline GO:0016054 & GO_Biological process & Organic acid catabolic process & $6.26 \times 10^{-9}$ & 16 \\
\hline GO:0008152 & GO_Biological process & Metabolic process & $1.29 \times 10^{-3}$ & 159 \\
\hline GO:0009056 & GO_Biological process & Catabolic process & $1.15 \times 10^{-1}$ & 28 \\
\hline GO:0007275 & GO_Biological process & Multicellular organismal development & $1.11 \times 10^{-6}$ & 102 \\
\hline GO:0048731 & GO_Biological process & System development & $9.71 \times 10^{-8}$ & 91 \\
\hline GO:0048519 & GO_Biological process & Negative regulation of biological process & $1.82 \times 10^{-5}$ & 72 \\
\hline GO:0048523 & GO_Biological process & Negative regulation of cellular process & $3.81 \times 10^{-5}$ & 66 \\
\hline GO:0050789 & GO_Biological process & Regulation of biological process & $3.62 \times 10^{-4}$ & 176 \\
\hline GO:0065007 & GO_Biological process & Biological regulation & $6.72 \times 10^{-5}$ & 189 \\
\hline GO:0008150 & GO_Biological process & Biological_process & $1.15 \times 10^{-3}$ & 336 \\
\hline GO:0032501 & GO_Biological process & Multicellular organismal process & $1.15 \times 10^{-5}$ & 133 \\
\hline GO:0044237 & GO_Biological process & Cellular metabolic process & $6.22 \times 10^{-3}$ & 132 \\
\hline GO:0034641 & GO_Biological process & Cellular nitrogen compound metabolic process & $2.19 \times 10^{-2}$ & 59 \\
\hline GO:0044238 & GO_Biological process & Primary metabolic process & $4.35 \times 10^{-3}$ & 140 \\
\hline GO:0050896 & GO_Biological process & Response to stimulus & $4.59 \times 10^{-5}$ & 112 \\
\hline GO:0009719 & GO_Biological process & Response to endogenous stimulus & $3.77 \times 10^{-9}$ & 34 \\
\hline GO:0050794 & GO_Biological process & Regulation of cellular process & $9.14 \times 10^{-4}$ & 166 \\
\hline GO:0023052 & GO_Biological process & Signaling & $5.63 \times 10^{-5}$ & 99 \\
\hline GO:0044281 & GO_Biological process & Small molecule metabolic process & $3.29 \times 10^{-8}$ & 62 \\
\hline GO:0006519 & GO_Biological process & Cellular amino acid and derivative metabolic process & $5.92 \times 10^{-5}$ & 21 \\
\hline GO:0043436 & GO_Biological process & Oxoacid metabolic process & $2.77 \times 10^{-9}$ & 37 \\
\hline GO:0019752 & GO_Biological process & Carboxylic acid metabolic process & $2.77 \times 10^{-9}$ & 37 \\
\hline GO:0009987 & GO_Biological process & Cellular process & $2.40 \times 10^{-8}$ & 258 \\
\hline GO:0046395 & GO_Biological process & Carboxylic acid catabolic process & $6.26 \times 10^{-9}$ & 16 \\
\hline GO:0009063 & GO_Biological process & Cellular amino acid catabolic process & $2.78 \times 10^{-6}$ & 10 \\
\hline GO:0044248 & GO_Biological process & Cellular catabolic process & $3.54 \times 10^{-2}$ & 25 \\
\hline GO:0009725 & GO_Biological process & Response to hormone stimulus & $4.39 \times 10^{-8}$ & 30 \\
\hline GO:0048545 & GO_Biological process & Response to steroid hormone stimulus & $2.46 \times 10^{-6}$ & 18 \\
\hline GO:0006807 & GO_Biological process & Nitrogen compound metabolic process & $2.95 \times 10^{-3}$ & 67 \\
\hline GO:0006520 & GO_Biological process & Cellular amino acid metabolic process & $4.99 \times 10^{-5}$ & 16 \\
\hline GO:0009605 & GO_Biological process & Response to external stimulus & $1.81 \times 10^{-8}$ & 35 \\
\hline GO:0009308 & GO_Biological process & Amine metabolic process & $4.15 \times 10^{-6}$ & 25 \\
\hline GO:0009310 & GO_Biological process & Amine catabolic process & $9.50 \times 10^{-6}$ & 10 \\
\hline GO:0032502 & GO_Biological process & Developmental process & $5.43 \times 10^{-7}$ & 110 \\
\hline GO:0048856 & GO_Biological process & Anatomical structure development & $8.77 \times 10^{-7}$ & 94 \\
\hline GO:0009653 & GO_Biological process & Anatomical structure morphogenesis & $1.19 \times 10^{-6}$ & 53 \\
\hline GO:0042221 & GO_Biological process & Response to chemical stimulus & $3.51 \times 10^{-10}$ & 70 \\
\hline GO:0010033 & GO_Biological process & Response to organic substance & $4.09 \times 10^{-11}$ & 52 \\
\hline GO:0010646 & GO_Biological process & Regulation of cell communication & $1.30 \times 10^{-5}$ & 48 \\
\hline GO:0008283 & GO_Biological process & Cell proliferation & $3.68 \times 10^{-6}$ & 26 \\
\hline GO:0044282 & GO_Biological process & Small molecule catabolic process & $2.35 \times 10^{-5}$ & 18 \\
\hline GO:0023046 & GO_Biological process & Signaling process & $8.53 \times 10^{-6}$ & 77 \\
\hline GO:0023060 & GO_Biological process & Signal transmission & $8.53 \times 10^{-6}$ & 77 \\
\hline GO:0042127 & GO_Biological process & Regulation of cell proliferation & $1.66 \times 10^{-6}$ & 41 \\
\hline GO:0048513 & GO_Biological process & Organ development & $5.37 \times 10^{-8}$ & 74 \\
\hline GO:0009888 & GO_Biological process & Tissue development & $2.37 \times 10^{-8}$ & 42 \\
\hline GO:0044106 & GO_Biological process & Cellular amine metabolic process & $1.70 \times 10^{-4}$ & 18 \\
\hline GO:0032787 & GO_Biological process & Monocarboxylic acid metabolic process & $3.81 \times 10^{-6}$ & 21 \\
\hline GO:0007165 & GO_Biological process & Signal transduction & $2.88 \times 10^{-6}$ & 71 \\
\hline GO:0010648 & GO_Biological process & Negative regulation of cell communication & $3.44 \times 10^{-6}$ & 22 \\
\hline GO:0051239 & GO_Biological process & Regulation of multicellular organismal process & $1.60 \times 10^{-6}$ & 48 \\
\hline
\end{tabular}


Table II. Continued.

\begin{tabular}{|c|c|c|c|c|}
\hline ID & Category & GO term & P-value & Count \\
\hline GO:0042180 & GO_Biological process & Cellular ketone metabolic process & $5.36 \times 10^{-9}$ & 37 \\
\hline GO:0050793 & GO_Biological process & Regulation of developmental process & $1.88 \times 10^{-6}$ & 39 \\
\hline GO:0005515 & GO_Molecular function & Protein binding & $2.06 \times 10^{-6}$ & 223 \\
\hline GO:0003674 & GO_Molecular function & Molecular_function & $4.03 \times 10^{-4}$ & 359 \\
\hline GO:0005488 & GO_Molecular function & Binding & $8.63 \times 10^{-8}$ & 316 \\
\hline GO:0048037 & GO_Molecular function & Cofactor binding & $3.31 \times 10^{-6}$ & 19 \\
\hline GO:0005102 & GO_Molecular function & Receptor binding & $1.45 \times 10^{-7}$ & 46 \\
\hline GO:0005575 & GO_Cellular component & Cellular_component & $1.14 \times 10^{-2}$ & 370 \\
\hline GO:0005615 & GO_Cellular component & Extracellular space & $1.96 \times 10^{-8}$ & 42 \\
\hline GO:0005576 & GO_Cellular component & Extracellular region & $1.10 \times 10^{-5}$ & 73 \\
\hline GO:0044421 & GO_Cellular component & Extracellular region part & $3.08 \times 10^{-9}$ & 52 \\
\hline
\end{tabular}

GO, Gene Ontology.

Table III. KEGG pathway analysis of the target genes of miR-338-5p from Cytoskape.

\begin{tabular}{|c|c|c|c|c|}
\hline ID & Name & Category & Term P-value & Count \\
\hline GO:0000280 & Valine, leucine and isoleucine degradation & KEGG & $5.1 \times 10^{-6}$ & 9 \\
\hline GO:0005200 & Pathways in cancer & KEGG & $9.5 \times 10^{-5}$ & 25 \\
\hline GO:0004110 & Cell cycle & KEGG & $1.4 \times 10^{-4}$ & 12 \\
\hline GO:0000071 & Fatty acid degradation & KEGG & $1.7 \times 10^{-4}$ & 7 \\
\hline GO:0000640 & Propanoate metabolism & KEGG & $2.0 \times 10^{-4}$ & 6 \\
\hline
\end{tabular}

KEGG, Kyoto Encyclopedia of Genes and Genomes.

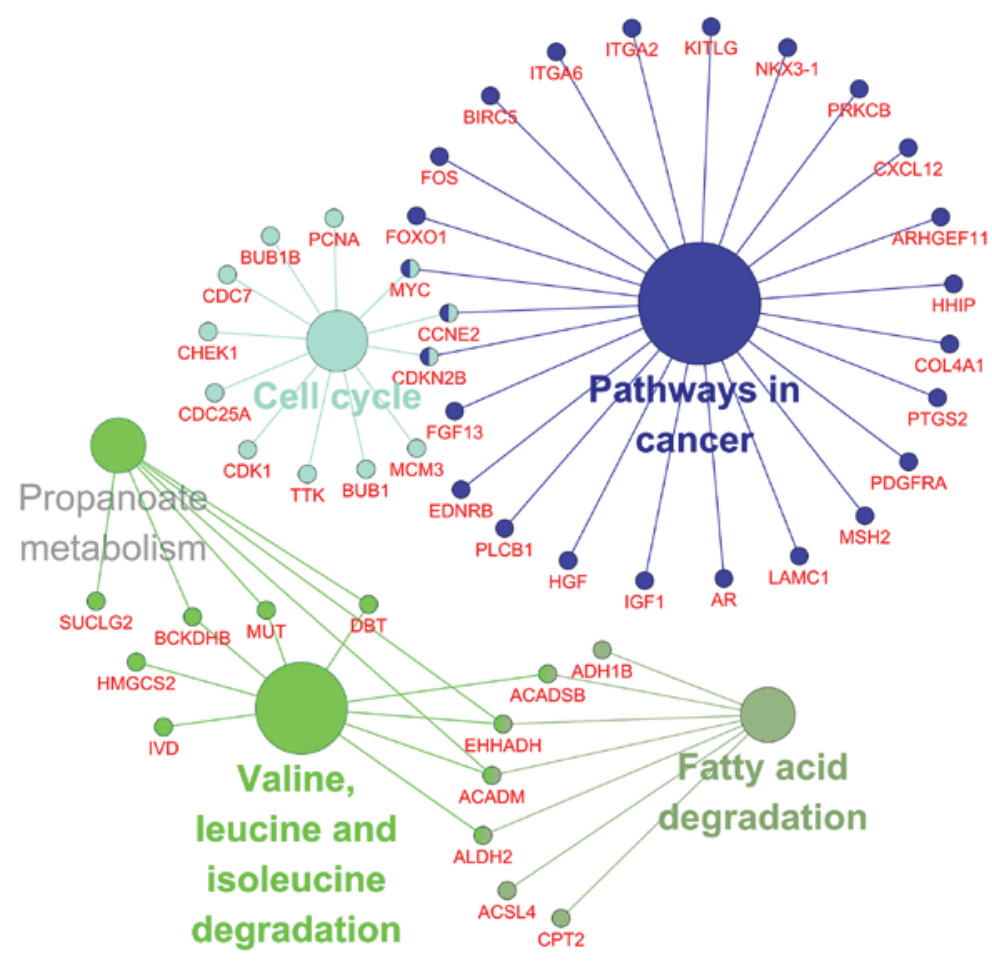

Figure 16. The network of KEGG pathway analysis. Five large circles in the network represented the corresponding significant pathways from KEGG. Small circles linked by the edges between the large circles symbolized the genes contained in these pathways and the common genes shared by multiple pathways were marked by a combination of colors. KEGG, Kyoto Encyclopedia of Genes and Genomes. 


\section{References}

1. Nault JC, De Reyniès A, Villanueva A, Calderaro J, Rebouissou S, Couchy G, Decaens T, Franco D, Imbeaud S, Rousseau F, et al: A hepatocellular carcinoma 5-gene score associated with survival of patients after liver resection. Gastroenterology 145: 176-187, 2013.

2. Chen W, Zheng R, Baade PD, Zhang S, Zeng H, Bray F, Jemal A, $\mathrm{Yu}$ XQ and He J: Cancer statistics in China, 2015. CA Cancer J Clin 66: 115-132, 2016

3. Zhu AX: Molecularly targeted therapy for advanced hepatocellular carcinoma in 2012: Current status and future perspectives. Semin Oncol 39: 493-502, 2012.

4. Llovet JM, Villanueva A, Lachenmayer A and Finn RS: Advances in targeted therapies for hepatocellular carcinoma in the genomic era. Nat Rev Clin Oncol 12: 408-424, 2015.

5. Lages E, Ipas H, Guttin A, Nesr H, Berger F and Issartel JP. MicroRNAs: Molecular features and role in cancer. Front Biosci (Landmark Ed) 17: 2508-2540, 2012.

6. Neilson JR and Sharp PA: Small RNA regulators of gene expression. Cell 134: 899-902, 2008

7. Volinia S, Calin GA, Liu CG, Ambs S, Cimmino A, Petrocca F Visone R, Iorio M, Roldo C, Ferracin M, et al: A microRNA expression signature of human solid tumors defines cancer gene targets. Proc Natl Acad Sci USA 103: 2257-2261, 2006.

8. Lee EJ, Gusev Y, Jiang J, Nuovo GJ, Lerner MR, Frankel WL, Morgan DL, Postier RG, Brackett DJ and Schmittgen TD: Expression profiling identifies microRNA signature in pancreatic cancer. Int J Cancer 120: 1046-1054, 2007.

9. Yanaihara N, Caplen N, Bowman E, Seike M, Kumamoto K, Yi M, Stephens RM, Okamoto A, Yokota J, Tanaka T, et al: Unique microRNA molecular profiles in lung cancer diagnosis and prognosis. Cancer Cell 9: 189-198, 2006

10. Murakami Y, Yasuda T, Saigo K, Urashima T, Toyoda H, Okanoue $\mathrm{T}$ and Shimotohno K: Comprehensive analysis of microRNA expression patterns in hepatocellular carcinoma and non-tumorous tissues. Oncogene 25: 2537-2545, 2006.

11. Calin GA, Liu CG, Sevignani C, Ferracin M, Felli N, Dumitru CD, Shimizu M, Cimmino A, Zupo S, Dono M, et al: MicroRNA profiling reveals distinct signatures in B cell chronic lymphocytic leukemias. Proc Natl Acad Sci USA 101: 11755-11760, 2004

12. Calin GA and Croce CM: MicroRNA signatures in human cancers. Nat Rev Cancer 6: 857-866, 2006

13. Tong D, Zhao L, He K, Sun H, Cai D, Ni L, Sun R, Chang S, Song T, Huang C, et al: MECP2 promotes the growth of gastric cancer cells by suppressing miR-338-mediated antiproliferative effect. Oncotarget 7: 34845-34859, 2016.

14. Chen JS, Liang LL, Xu HX, Chen F, Shen SL, Chen W, Chen LZ, Su Q, Zhang LJ, Bi J, et al: miR-338-3p inhibits epithelial-mesenchymal transition and metastasis in hepatocellular carcinoma cells. Oncotarget 8: 71418-71429, 2016.

15. Chen X, Wei L and Zhao S: miR-338 inhibits the metastasis of lung cancer by targeting integrin $\beta 3$. Oncol Rep 36: 1467-1474, 2016.

16. Weng HL and Wang MJ: Effects of microRNA-338-3p on morphine-induced apoptosis and its underlying mechanisms. Mol Med Rep 14: 2085-2092, 2016.

17. Zhuang Y, Dai J, Wang Y, Zhang H, Li X, Wang C, Cao M, Liu Y, Cai H, Zhang D and Wang Y: miR-338* suppresses fibrotic pathogenesis in pulmonary fibrosis through targeting LPA1. Am J Transl Res 8: 3197-3205, 2016.

18. Xing Z, Yu L, Li X and Su X: Anticancer bioactive peptide-3 inhibits human gastric cancer growth by targeting miR-338-5p. Cell Biosci 6: 53, 2016.

19. Yong FL, Law CW and Wang CW: Potentiality of a triple microRNA classifier: miR-193a-3p, miR-23a and miR-338-5p for early detection of colorectal cancer. BMC Cancer 13: 280, 2013.

20. Besse A, Sana J, Lakomy R, Kren L, Fadrus P, Smrcka M, Hermanova M, Jancalek R, Reguli S, Lipina R, et al: miR-338-5p sensitizes glioblastoma cells to radiation through regulation of genes involved in DNA damage response. Tumour Biol 37: 7719-7727, 2016

21. Chen Y, Chen J, Liu Y, Li S and Huang P: Plasma miR-15b-5p, miR-338-5p, and miR-764 as biomarkers for hepatocellular carcinoma. Med Sci Monit 21: 1864-1871, 2015.

22. Deeks JJ, Macaskill P and Irwig L: The performance of tests of publication bias and other sample size effects in systematic reviews of diagnostic test accuracy was assessed. J Clin Epidemiol 58: 882-893, 2005.
23. Glas AS, Lijmer JG, Prins MH, Bonsel GJ and Bossuyt PM: The diagnostic odds ratio: A single indicator of test performance. J Clin Epidemiol 56: 1129-1135, 2003.

24. Harbord RM, Deeks JJ, Egger M, Whiting P and Sterne JA: A unification of models for meta-analysis of diagnostic accuracy studies. Biostatistics 8: 239-251, 2007.

25. Higgins JP, Thompson SG, Deeks JJ and Altman DG: Measuring inconsistency in meta-analyses. BMJ 327: 557-560, 2003.

26. Jackson D, White IR and Thompson SG: Extending DerSimonian and Laird's methodology to perform multivariate random effects meta-analyses. Stat Med 29: 1282-1297, 2010.

27. Uhlen M, Oksvold P, Fagerberg L, Lundberg E, Jonasson K, Forsberg M, Zwahlen M, Kampf C, Wester K, Hober S, et al: Towards a knowledge-based Human protein atlas. Nat Biotechnol 28: 1248-1250, 2010.

28. Chen Y, Huang P, Chen J, Liu Y, Li SL, Wang Z and Song D: Plasma circulating miR-338-5p, miR-21-5p and miR-15b-5p are potential biomarkers for screening hepatocellular carcinoma. J Third Mil Med Univ 37: 1720-1726, 2015 (In Chinese).

29. Budhu A, Jia HL, Forgues M, Liu CG, Goldstein D, Lam A, Zanetti KA, Ye QH, Qin LX, Croce CM, et al: Identification of metastasis-related microRNAs in hepatocellular carcinoma. Hepatology 47: 897-907, 2008.

30. Su H, Yang JR, Xu T, Huang J, Xu L, Yuan Y and Zhuang SM: MicroRNA-101, down-regulated in hepatocellular carcinoma, promotes apoptosis and suppresses tumorigenicity. Cancer Res 69: 1135-1142, 2009.

31. Burchard J, Zhang C, Liu AM, Poon RT, Lee NP, Wong KF, Sham PC,Lam BY, Ferguson MD, Tokiwa G, et al: microRNA-122 as a regulator of mitochondrial metabolic gene network in hepatocellular carcinoma. Mol Syst Biol 6: 402, 2010.

32. Diaz G, Melis M, Tice A, Kleiner DE, Mishra L, Zamboni F and Farci P: Identification of microRNAs specifically expressed in hepatitis C virus-associated hepatocellular carcinoma. Int J Cancer 133: 816-824, 2013

33. Shen J, Wang A, Wang Q, Gurvich I, Siegel AB, Remotti H and Santella RM: Exploration of genome-wide circulating microRNA in hepatocellular carcinoma: $\operatorname{miR}-483-5 p$ as a potential biomarker. Cancer Epidemiol Biomarkers Prev 22: 2364-2373, 2013.

34. Murakami Y, Kubo S, Tamori A, Itami S, Kawamura E, Iwaisako K, Ikeda K, Kawada N, Ochiya T and Taguchi YH: Comprehensive analysis of transcriptome and metabolome analysis in Intrahepatic cholangiocarcinoma and hepatocellular carcinoma. Sci Rep 5: 16294, 2015.

35. Martinez-Quetglas I, Pinyol R, Dauch D, Torrecilla S, Tovar V, Moeini A, Alsinet C, Portela A, Rodriguez-Carunchio L, Solé M, et al: IGF2 is up-regulated by epigenetic mechanisms in hepatocellular carcinomas and is an actionable oncogene product in experimental models. Gastroenterology 151: 1192-1205, 2016

36. Shih TC, Tien YJ, Wen CJ, Yeh TS, Yu MC, Huang CH, Lee YS, Yen TC and Hsieh SY: MicroRNA-214 downregulation contributes to tumor angiogenesis by inducing secretion of the hepatoma-derived growth factor in human hepatoma. J Hepatol 57: 584-591, 2012.

37. Noh JH, Chang YG, Kim MG, Jung KH, Kim JK, Bae HJ, Eun JW, Shen Q, Kim SJ, Kwon SH, et al: miR-145 functions as a tumor suppressor by directly targeting histone deacetylase 2 in liver cancer. Cancer Lett 335: 455-462, 2013.

38. Sato F, Hatano E, Kitamura K, Myomoto A, Fujiwara T, Takizawa S, Tsuchiya S, Tsujimoto G, Uemoto S and Shimizu K: MicroRNA profile predicts recurrence after resection in patients with hepatocellular carcinoma within the Milan Criteria. PLoS One 6: e16435, 2011.

39. Zhang Y, Li T, Qiu Y, Zhang T, Guo P, Ma X, Wei Q and Han L: Serum microRNA panel for early diagnosis of the onset of hepatocellular carcinoma. Medicine (Baltimore) 96: e5642, 2017.

40. Okajima W, Komatsu S, Ichikawa D, Miyamae M, Kawaguchi T, Hirajima S, Ohashi T, Imamura T, Kiuchi J, Arita T, et al: Circulating microRNA profiles in plasma: Identification of miR-224 as a novel diagnostic biomarker in hepatocellular carcinoma independent of hepatic function. Oncotarget 7 : 53820-53836, 2016.

41. Yan SR, Liu ZJ, Yu S and Bao YX: Investigation of the value of miR-21 in the diagnosis of early stage HCC and its prognosis: A meta-analysis. Genet Mol Res 14: 11573-11586, 2015.

42. Lei D, Zhang F, Yao D, Xiong N, Jiang X and Zhao H: miR-338-5p suppresses proliferation, migration, invasion, and promote apoptosis of glioblastoma cells by directly targeting EFEMP1. Biomed Pharmacother 89: 957-965, 2017. 
43. Yang W, Cho H, Shin HY, Chung JY, Kang ES, Lee EJ and Kim JH: Accumulation of cytoplasmic Cdk1 is associated with cancer growth and survival rate in epithelial ovarian cancer. Oncotarget 7: 49481-49497, 2016.

44. Tsaur I, Makarević J, Hudak L, Juengel E, Kurosch M, Wiesner C, Bartsch G, Harder S, Haferkamp A and Blaheta RA: The cdk1-cyclin B complex is involved in everolimus triggered resistance in the PC3 prostate cancer cell line. Cancer Lett 313: 84-90, 2011.

45. Sung WW, Lin YM, Wu PR, Yen HH, Lai HW, Su TC, Huang RH, Wen CK, Chen CY, Chen CJ and Yeh KT: High nuclear/cytoplasmic ratio of Cdk1 expression predicts poor prognosis in colorectal cancer patients. BMC Cancer 14: 951, 2014.

46. Willder JM, Heng SJ, McCall P, Adams CE, Tannahill C, Fyffe G, Seywright M, Horgan PG, Leung HY, Underwood MA and Edwards J: Androgen receptor phosphorylation at serine 515 by Cdk1 predicts biochemical relapse in prostate cancer patients. Br J Cancer 108: 139-148, 2013.

47. Banerjee SK, Weston AP, Zoubine MN, Campbell DR and Cherian R: Expression of cde2 and cyclin B1 in Helicobacter pylori-associated gastric MALT and MALT lymphoma: Relationship to cell death, proliferation, and transformation. Am J Pathol 156: 217-225, 2000.

48. Hongo F, Takaha N, Oishi M, Ueda T, Nakamura T, Naitoh Y, Naya Y, Kamoi K, Okihara K, Matsushima T, et al: CDK1 and CDK2 activity is a strong predictor of renal cell carcinoma recurrence. Urol Oncol 32: 1240-1246, 2014.

49. Tian J, Hu X, Gao W, Zhang J, Chen M, Zhang X, Ma J and Yuan H: Identification a novel tumor-suppressive hsa-miR-599 regulates cells proliferation, migration and invasion by targeting oncogenic MYC in hepatocellular carcinoma. Am J Transl Res 8: 2575-2584, 2016.

50. Cao L, Li C, Shen S, Yan Y, Ji W, Wang J, Qian H, Jiang X, Li Z, Wu M, et al: OCT4 increases BIRC5 and CCND1 expression and promotes cancer progression in hepatocellular carcinoma. BMC Cancer 13: 82, 2013.

51. Le Coz V, Zhu C, Devocelle A, Vazquez A, Boucheix C, Azzi S, Gallerne C, Eid P, Lecourt S and Giron-Michel J: IGF-1 contributes to the expansion of melanoma-initiating cells through an epithelial-mesenchymal transition process. Oncotarget 7: 82511-82527, 2016.

52. Yang XW, Shen GZ, Cao LQ, Jiang XF, Peng HP, Shen G, Chen D and Xue P: MicroRNA-1269 promotes proliferation in human hepatocellular carcinoma via downregulation of FOXO1. BMC Cancer 14: 909, 2014.
53. Ko YS, Cho SJ, Park J, Kim Y, Choi YJ, Pyo JS, Jang BG, Park JW, Kim WH and Lee BL: Loss of FOXO1 promotes gastric tumour growth and metastasis through upregulation of human epidermal growth factor receptor $2 /$ neu expression. Br J Cancer 113: 1186-1196, 2015.

54. Ren JW, Li ZJ and Tu C: miR-135 post-transcriptionally regulates FOXO1 expression and promotes cell proliferation in human malignant melanoma cells. Int J Clin Exp Pathol 8: 6356-6366, 2015.

55. Martinez-Iglesias OA, Alonso-Merino E, Gómez-Rey S, Velasco-Martín JP, Martín Orozco R, Luengo E, García Martín R, Ibáñez de Cáceres I, Fernández AF, Fraga MF, et al: Autoregulatory loop of nuclear corepressor 1 expression controls invasion, tumor growth, and metastasis. Proc Natl Acad Sci USA 113: E328-E337, 2016.

56. Yu JJ, Wu YX, Zhao FJ and Xia SJ: miR-96 promotes cell proliferation and clonogenicity by down-regulating of FOXO1 in prostate cancer cells. Med Oncol 31: 910, 2014.

57. Zhang B, Gui LS, Zhao XL, Zhu LL and Li QW: FOXO1 is a tumor suppressor in cervical cancer. Genet Mol Res 14: 6605-6616, 2015.

58. Li K, Chen MK, Situ J, Huang WT, Su ZL, He D and Gao X: Role of co-expression of c-Myc, EZH2 and p27 in prognosis of prostate cancer patients after surgery. Chin Med J (Engl) 126: 82-87, 2013.

59. Liu Z, Jiang Y, Hou Y, Hu Y, Cao X, Tao Y, Xu C, Liu S, Wang S, Wang L, et al: The IкB family member Bcl-3 stabilizes c-Myc in colorectal cancer. J Mol Cell Biol 5: 280-282, 2013.

60. Duffy MJ, O'Donovan N, Brennan DJ, Gallagher WM and Ryan BM: Survivin: A promising tumor biomarker. Cancer Lett 249: 49-60, 2007.

61. Grimberg A: Mechanisms by which IGF-I may promote cancer. Cancer Biol Ther 2: 630-635, 2003.

62. Xie L, Ushmorov A, Leithäuser F, Guan H, Steidl C, Färbinger J, Pelzer C, Vogel MJ, Maier HJ, Gascoyne RD, et al: FOXO1 is a tumor suppressor in classical Hodgkin lymphoma. Blood 119: 3503-3511, 2012.

63. Chun YS, Huang M, Rink L and Von Mehren M: Expression levels of insulin-like growth factors and receptors in hepatocellular carcinoma: A retrospective study. World J Surg Oncol 12: 231, 2014.

This work is licensed under a Creative Commons Attribution-NonCommercial-NoDerivatives 4.0 International (CC BY-NC-ND 4.0) License. 